\title{
Unique prediction of cannabis use severity and behaviors by delay discounting and behavioral economic demand
}

\author{
Justin C. Strickland ${ }^{\mathrm{a}, *}$, Joshua A. Lile ${ }^{\mathrm{a}, \mathrm{b}, \mathrm{c}}$, William W. Stoops ${ }^{\mathrm{a}, \mathrm{b}, \mathrm{c}}$ \\ a Department of Psychology, University of Kentucky College of Arts and Sciences, 171 Funkhouser Drive, Lexington, KY 40506-0044, USA \\ b Department of Behavioral Science, University of Kentucky College of Medicine, 1100 Veterans Drive, Medical Behavioral Science Building Room 140, Lexington, KY \\ 40536-0086, USA \\ ${ }^{c}$ Department of Psychiatry, University of Kentucky College of Medicine, 3470 Blazer Parkway, Lexington, KY 40509-1810, USA
}

\section{A R T I C L E I N F O}

\section{Keywords:}

Behavioral economics

Crowdsourcing

Demand curve

Drug

Substance use

\begin{abstract}
A B S T R A C T
Few studies have simultaneously evaluated delay discounting and behavioral economic demand to determine their unique contribution to drug use. A recent study in cannabis users found that monetary delay discounting uniquely predicted cannabis dependence symptoms, whereas cannabis demand uniquely predicted use frequency. This study sought to replicate and extend this research by evaluating delay discounting and behavioral economic demand measures for multiple commodities and including a use quantity measure. Amazon.com's Mechanical Turk was used to sample individuals reporting recent cannabis use ( $n=64)$ and controls $(\mathrm{n}=72)$. Participants completed measures of monetary delay discounting as well as alcohol and cannabis delay discounting and demand. Cannabis users and controls did not differ on monetary delay discounting or alcohol delay discounting and demand. Among cannabis users, regression analyses indicated that cannabis delay discounting uniquely predicted use severity, whereas cannabis demand uniquely predicted use frequency and quantity. These effects remained significant after controlling for other delay discounting and demand measures. This research replicates previous outcomes relating delay discounting and demand with cannabis use and extends them by accounting for the contribution of multiple commodities. This research also demonstrates the ability of online crowdsourcing methods to complement traditional human laboratory techniques.
\end{abstract}

\section{Introduction}

The marriage of behavioral economics with substance use research over the last three decades has advanced an understanding of the etiology and treatment of drug-taking behavior (Bickel et al., 2000; Chivers and Higgins, 2012; Hursh, 1984; MacKillop, 2016). Two of the most common applications of behavioral economics to drug use are delay discounting and behavioral economic demand. Delay discounting is the systematic reduction in value of a reinforcer as a function of the delay to its delivery (Green and Myerson, 2004; Odum, 2011; Rachlin and Green, 1972). Excessive delay discounting is thought central to substance use disorders and may represent a trans-disease process relating drug use to other maladaptive health behaviors (Bickel et al., 2012; Koffarnus et al., 2013). Several meta-analyses support this assertion by demonstrating a robust relationship between delayed reward discounting and drug use severity, dependence, and quantityfrequency variables (Amlung et al., 2017; MacKillop et al., 2011). This literature also provides evidence for an association between delay discounting and treatment response across diverse clinical populations (e.g., Krishnan-Sarin et al., 2007; MacKillop and Kahler, 2009; Washio et al., 2011; Yoon et al., 2007).

Substance use researchers have also situated drug use within a commodity purchase framework and used behavioral economic demand models to describe drug-taking behavior (e.g., Hursh, 1984, 1993; Johnson and Bickel, 2006). A recent popular extension of these methods is the commodity purchase task in which participants report hypothetical or realized commodity consumption across a range of prices per unit of the commodity (Jacobs and Bickel, 1999; MacKillop et al., 2008; Murphy et al., 2009). To date, purchase tasks have been successfully applied to a variety of drugs and drug classes, including alcohol, cannabis, cigarettes, cocaine, opioids, and synthetic cathinones (Amlung and MacKillop, 2015; Aston et al., 2015, 2016; Bruner and Johnson, 2014; Collins et al., 2014; Johnson and Johnson, 2014; MacKillop et al., 2008; Murphy and MacKillop, 2006; Pickover et al., 2016). These studies have demonstrated that commonly used and misused substances follow the same prototypic patterns of consumption

\footnotetext{
* Corresponding author.

E-mail address: justrickland@uky.edu (J.C. Strickland).
} 
as other goods, including decreases in consumption with increases in price, and price ranges at which consumption is sensitive (i.e., elastic) or insensitive (i.e., inelastic) to price change. Such research has also helped reveal behavioral mechanisms by which putative interventions may decrease drug consumption (Bujarski et al., 2012; McClure et al., 2013) and prognostic variables predicting treatment success (MacKillop and Murphy, 2007; Murphy et al., 2015).

Despite being the most widely used illicit substance in the United States, cannabis has received comparatively little attention in the delay discounting and demand literatures. Further information on cannabis delay discounting and demand could be useful given the increasing number of states proposing or that have passed legalized recreational use, and the growing prevalence of cannabis use reported in the United States over the last decade (Center for Behavioral Health Statistics, 2016). Although many first-time or recreational users will not continue to regular use, others will progress to problematic usage patterns and seek treatment for cannabis use disorder. The application of behavioral economic theory could help identify behavioral mechanisms contributing to maladaptive use and hasten the design of preventative and therapeutic interventions.

The association between delay discounting and cannabis use is more variable than for other drugs. For example, several studies have failed to show significant differences in delay discounting rates between cannabis users and controls (Johnson et al., 2010) or a relationship between delay discounting and treatment outcomes (Heinz et al., 2013; Peters et al., 2013; but see Stanger et al., 2012). However, others have demonstrated a role for delay discounting in aspects of cannabis use with significant associations observed between delay discounting and cannabis use initiation and severity (Aston et al., 2016; Bidwell et al., 2013; Heinz et al., 2013; Kollins, 2003). These differences in experimental outcomes could stem from the variations in the questions posed, namely delay discounting in cannabis users compared to controls as opposed to correlations between delay discounting and features of cannabis use (e.g., use severity). This limited literature also displays heterogeneity with respect to sample characteristics (e.g., college students versus treatment samples), delay discounting measures, and the commodity discounted. Such heterogeneity underscores the need for additional research to evaluate the association between delay discounting and aspects of cannabis use, and to compare delay discounting rates between cannabis users and controls.

Cannabis demand has received even less attention than delay discounting, representing a research literature in its infancy. The few extant studies have revealed outcomes consistent with previous work with other drugs, such as a sensitivity of cannabis demand to increased cost and an expected relationship between cannabis demand and measures of cannabis use severity and frequency (Aston et al., 2015; Collins et al., 2014). These studies have also demonstrated changes in cannabis demand following manipulations affecting state disposition, with increases observed in cannabis demand after cannabis-cue presentation (Metrik et al., 2016).

Recent attempts have been made to unify the ideas of delay discounting and demand under a broader "reinforcer pathology" conceptualization of substance use (Bickel et al., 2011). This approach posits that substance use disorders are characterized by an extreme preference for immediate consumption of a drug reinforcer (i.e., delay discounting) combined with high valuation of that reinforcer (i.e., behavioral economic demand). Relatively little research, however, has simultaneously evaluated demand and delay discounting metrics despite their mutual importance for this and other theoretical models. In fact, only one study has done so in the context of cannabis use (Aston et al., 2016). Data in that study were combined from participants completing a delay discounting task for money following placebo smoked cannabis administration and a purchase task for hypothetical cannabis completed during a baseline screening session. Delay discounting and behavioral economic demand functioned as independent predictors, with monetary delay discounting uniquely predicting can- nabis dependence (CD) symptom count and cannabis demand uniquely predicting frequency of cannabis use. However, this previous study only evaluated monetary delay discounting and cannabis demand. The inclusion of delay discounting and demand measures for multiple drug commodities (e.g., cannabis, alcohol) would help to demonstrate the specificity of cannabis-relevant outcomes for predicting use behaviors.

The purpose of the present study was to replicate and extend those findings relating delay discounting and demand to cannabis use behaviors. To this end, Amazon.com's Mechanical Turk (mTurk) was used to sample individuals reporting recent cannabis use. This study sought to extend previous research by 1) using an alternative sampling method allowing for greater demographic and drug use variability, 2) evaluating multiple commodities for delay discounting and demand variables, and 3) including a measure of use quantity (i.e., grams used per week) to further describe cannabis use patterns. Cannabis delay discounting was expected to uniquely predict $\mathrm{CD}$ symptom count and cannabis demand was expected to uniquely predict frequency and quantity of cannabis use. Alcohol delay discounting and demand were not expected to relate to cannabis use behaviors. As a secondary analysis, measures of delay discounting and behavioral economic demand were compared between cannabis users and non-cannabis using controls to add to the limited literature evaluating these outcomes between these groups. Significant differences between cannabis users and controls were not expected.

\section{Methods}

\subsection{Participants and procedures}

Participants were sampled using mTurk where tasks are advertised as Human Intelligence Tasks (HITs). Participants were required to have a $95 \%$ or higher approval rating on all previously submitted mTurk HITs, over 100 approved HITs, and current residence in the United States to view the study HIT. The accuracy of these inclusion criteria was verified by the mTurk platform. Participants reviewed an informed consent document describing the study procedures, compensation, and the fact that anonymity would be retained throughout the study. All respondents indicated by electronic confirmation that they understood this document and agreed to participate. The University of Kentucky Institutional Review Board approved all protocols, including the consent process, and the protocol was carried out in accordance with the Declaration of Helsinki.

Participants completed the study tasks as a part of larger study on choice and decision-making. A short screening questionnaire was used to determine if participants qualified for this study. Participants were only able to complete the screener once. Eligible participants were individuals reporting cannabis use during the past two weeks and 50 or more lifetime uses $(n=78)$. An additional control group was used to compare behavioral economic demand and delay discounting outcomes and included participants who did not report cannabis use in the past two weeks and five or fewer lifetime uses $(n=86)$. All participants were 18 years of age or older. Four attention checks were used to identify non-systematic, inattentive, or inconsistent participant data: 1) comparison of age and sex responses at the start and end of the survey, 2) recall at the end of the survey of a single digit number presented halfway through that participants were instructed to remember, 3) an item that instructed participants to select a specific response (i.e., "Select 'A Little Bit"), and 4) an item that asked participants if they had been attentive and thought their data should be included. Participants failing one or more checks were removed from data analysis, which resulted in a final sample size of 136 participants (cannabis users $=64$; controls $=72$ ). Demographic and drug use variables for cannabis users and controls are presented in Table 1. 
Table 1

Demographics and Drug Use Variables.

\begin{tabular}{|c|c|c|c|}
\hline & \multirow{2}{*}{$\begin{array}{l}\text { Cannabis User } \\
(\mathrm{n}=64) \\
\text { Mean (SD)/\% }\end{array}$} & \multicolumn{2}{|c|}{ Control $(\mathrm{n}=72)$} \\
\hline & & Mean (SD) $/ \%$ & $p$ \\
\hline \multicolumn{4}{|l|}{ Demographics } \\
\hline Age & $30.2(7.3)$ & $33.7(9.9)$ & $0.02^{*}$ \\
\hline Male & $48.4 \%$ & $44.4 \%$ & 0.73 \\
\hline White & $74.2 \%$ & $75.0 \%$ & 0.99 \\
\hline College Education & $50.0 \%$ & $65.3 \%$ & 0.08 \\
\hline Household Income & $\$ 41,094(\$ 28,122)$ & $\begin{array}{l}\$ 43,889 \\
(\$ 28,756)\end{array}$ & 0.57 \\
\hline \multicolumn{4}{|l|}{ Cannabis Use } \\
\hline Ever Use & $100.0 \%$ & $34.7 \%$ & - \\
\hline Lifetime Use & $5810(21,166)$ & $0.8(1.7)$ & - \\
\hline \% Month Use Days & $63.7 \%(34.4 \%)$ & $0 \%$ & - \\
\hline Grams/Week & $6.4(7.1)$ & - & - \\
\hline \# CD Symptoms & $2.0(2.1)$ & - & - \\
\hline \multicolumn{4}{|l|}{ Other Drug Use } \\
\hline CIG Use & $60.9 \%$ & $15.3 \%$ & $0.001^{\text {*k*n }}$ \\
\hline FTND $^{\mathrm{a}}$ & $3.9(2.6)$ & $4.0(3.0)$ & 0.88 \\
\hline ALC Use & $85.9 \%$ & $59.7 \%$ & $0.001^{* * *}$ \\
\hline Drinks/Week ${ }^{\mathrm{b}}$ & $9.2(14.4)$ & $3.7(7.8)$ & $0.03^{*}$ \\
\hline Drinking Days/Week ${ }^{\mathrm{b}}$ & $2.5(2.0)$ & $1.6(1.4)$ & $0.01^{*}$ \\
\hline Heavy Use Day ${ }^{\mathrm{b}}$ & $72.7 \%$ & $41.9 \%$ & $0.003^{\text {*.* }}$ \\
\hline
\end{tabular}

Note. CIG Use = cigarette use in the past two weeks determined from study screener; ALC Use $=$ alcohol use in the past two weeks determined from study screener; \# CD Symptoms $=$ number of cannabis use dependence symptoms endorsed; FTND $=$ Fagerström Test for Nicotine Dependence; Heavy Use Day $=$ reported past month heavy alcohol use day ( 5 or more [males] or 4 or more [females] drinks in a single day).

${ }^{\text {a }}$ Variable only for participants reporting cigarette use in the past two weeks ( $\mathrm{n}=39$ Cannabis Users; $\mathrm{n}=11$ Controls).

${ }^{\mathrm{b}}$ Variable only for participants reporting alcohol use in the past two weeks ( $\mathrm{n}=55$ Cannabis Users; $\mathrm{n}=43$ Controls).

$* p<0.05$.

$* * p<0.01$

$* * * p<0.001$.

\subsection{Measures}

\subsubsection{5-trial adjusting delay task}

A 5-trial adjusting delay task was used to evaluate delay discounting rates (for details see Koffarnus and Bickel, 2014). This task has been previously validated against traditional adjusting amount delay discounting tasks (Cox and Dallery, 2016; Koffarnus and Bickel, 2014). Participants were asked to select between some amount of a delayed commodity and half that amount available immediately. The delayed and immediate amounts remained constant while the delay to the larger amount was adjusted after each choice. The first choice was always at a three-week delay, which then adjusted up (longer delay following delayed choice) or down (shorter delay following immediate choice) based on decisions. The $\mathrm{ED}_{50}$, the inverse of the delay discounting rate or $k$, was determined following five choices and included 32 potential values between $1 \mathrm{~h}$ and 25 years. The benefits of this 5 -trial task include rapid assessment of delay discounting rates and minimal computing requirements. These advantages are particularly important given the online research context wherein time is limited and data collection is constrained by the participant's computer equipment.

Participants completed up to three different versions of the task. All participants completed a traditional monetary delay task, with $\$ 1000$ available delayed versus $\$ 500$ available now. All cannabis-using participants also completed a cannabis delay discounting task, with 1 ounce of typical quality cannabis available delayed versus $1 / 2$ ounce available now. Any participant endorsing current alcohol use also completed an alcohol delay task, with 24 US standard drinks available delayed versus 12 drinks available now (standard drink: one $12 \mathrm{oz}$. beer, $5 \mathrm{oz}$. wine, or $1.5 \mathrm{oz}$. shot/mixed drink). These commodity amounts were within ranges used in previous alcohol and cannabis delay discounting studies (Johnson et al., 2010; Petry, 2001). All delay discounting tasks included a statement emphasizing that consumption was not constrained by time and that the hypothetical goods could be kept.

\subsubsection{Commodity purchase tasks}

Commodity purchase tasks were used to evaluate behavioral economic demand for cannabis and alcohol. The same scenario was used in each task and all purchasing situations were framed as hypothetical in the present tense. Participants were asked to imagine a typical day over the last month when they used the commodity. They were told that they could only get the commodity from this source, had no commodity saved or kept from previous days, could not stockpile, and would have to consume all purchases in a single day. Participants were then asked how many drinks (alcohol) or hits (cannabis) they would purchase at 13 monetary increments ranging from $\$ 0.00$ [free] to $\$ 11 /$ unit, presented sequentially. This price range was selected due to its similarity to price ranges used in recent commodity purchase task studies (Amlung et al., 2015a; Aston et al., 2015, 2016; Murphy et al., 2015). Alcohol drinks were described as one US standard drink. Cannabis hits were quantified as $0.09 \mathrm{~g}$ of average quality cannabis (i.e., 10 hits $=1$ joint or $0.9 \mathrm{~g}$ or $1 / 32$ nd of an ounce) consistent with previous literature (Aston et al., 2015). All cannabis-using participants completed the commodity purchase task for cannabis (Marijuana Purchase Task [MPT]; note the term Marijuana Purchase Task [MPT] is used to avoid confusion with the commonly used Cigarette Purchase Task [CPT]) and all participants endorsing current alcohol use the task for alcohol (Alcohol Purchase Task; APT).

\subsubsection{Drug use variables}

A written version of the Mini-International Neuropsychiatric Interview (MINI) was used to evaluate cannabis dependence (CD) through endorsement of statements indicative of DSM-IV criteria (Sheehan et al., 1998). A Cannabis History and Smoking Questionnaire included questions about age of use onset, cannabis use patterns, and routes of administration (e.g., Aston et al., 2015; Metrik et al., 2009). Cannabis use variables included use severity (number of DSM-IV CD symptoms endorsed), frequency (percentage past month use days), and quantity (grams used per week), consistent with previous delay and demand literature (Aston et al., 2016). Other relevant drug use variables and demographics, such as age and the Fagerström Test for Nicotine Dependence (FTND), were also measured.

\subsection{Data analysis}

Delay discounting rates ( $k$ values) were calculated by taking the inverse of $\mathrm{ED}_{50}$ values derived from the 5-trial adjusting delay task (Koffarnus and Bickel, 2014). Delay discounting rates were log-transformed prior to analysis to obtain normality. Demand curves were first evaluated for inattentive data or non-systematic curves using standard criteria (see details in Stein et al., 2015). Briefly, curves were evaluated for increased consumption with increased price, frequent price-to-price consumption increases, or reversals from zero consumption as well as extreme consumption (i.e., greater than 100 drinks or hits [9 $\mathrm{g}$ of cannabis] in one day). Nine cannabis curves (14.1\%) and 16 alcohol curves $(15.7 \%)$ were determined non-systematic/inattentive and removed from demand analysis. Price elasticity and intensity were generated using the exponentiated demand equation:

$Q=Q_{0} * 10^{k *\left(e^{\left(-\alpha * Q_{0} * C\right)}-1\right)}$

where $Q=$ consumption; $Q_{0}=$ derived intensity of demand (consumption at zero price); $k=\mathrm{a}$ constant that denotes consumption range in $\log$ units ( $a$ priori set to 2); $C=$ the price of the commodity; and $\alpha=$ derived elasticity of demand. The exponentiated model is a recently developed and validated equation that allows for the inclusion 
of zero consumption values (Koffarnus et al., 2015; Strickland et al., 2016b). Area under the curve (AUC) values were also generated as described previously (Amlung et al., 2015b; Aston et al., 2016). Briefly, the total area was operationalized as the AUC value when the maximum consumption value across the sample was inputted for each price (100 for both the MPT and APT). Proportionate AUC values were then generated by dividing each participants raw AUC by this total AUC (range $=0.0-1.0$ ). Recent reports have proposed AUC as a single demand metric that is useful and valid measure to minimize repeated testing with multiple demand metrics and to allow for convergence with other behavioral economic measures (Amlung et al., 2015b; Aston et al., 2016). All demand metrics were log-transformed to correct for skew.

Demographic and drug use variables for cannabis users and controls were compared using independent sample $t$-tests. Independent sample $t$ tests were also used to compare monetary delay discounting as well as alcohol delay discounting and demand between groups. The relationship between cannabis use and demographic, demand, and delay discounting variables was first described using bivariate correlations in the cannabis-using group. The independent contribution of demand and delay discounting for predicting cannabis use was then determined using multiple regression models. Consistent with previous studies (Aston et al., 2016), AUC was first used to quantify demand in these analyses. Follow up analyses were then conducted using demand intensity and elasticity in place of AUC. Additional tests were also conducted controlling for age, sex, income, and cigarette use (given the close association between cigarette use and delay discounting; Bickel et al., 1999; Johnson et al., 2007). Only cannabis-using participants with all demand and delay discounting variables were included in regression analysis ( $\mathrm{n}=46$ with complete data). All tests were conducted using SPSS 24 and GraphPad Prism 6.0 with a type I error rate of 0.05 .

\section{Results}

\subsection{Between-group comparisons}

\subsubsection{Demographics and drug use}

Individuals reporting cannabis use were younger and more likely to report recent cigarette and alcohol use (i.e., in the past two weeks). Cannabis users also reported more drinking days per week and alcoholic drinks per week. Other demographic variables (e.g., race, income) did not differ between groups (Table 1).

\subsubsection{Delay discounting}

Raw monetary, cannabis, and alcohol delay discounting rates are presented in Table 2. Monetary delay discounting did not differ between cannabis users and control $\left(t_{134}=0.52, p=0.60\right)$. Similarly, alcohol delay discounting did not differ between these groups $\left(t_{100}=0.38, p=0.70\right)$. No differences in the magnitude or significance of these findings were observed after controlling for relevant covariates (e.g., age, cigarette use, alcohol use).

\subsubsection{Drug demand}

Demand curves for the MPT and APT showed prototypical decreases in consumption with increases in price for both groups (Fig. 1). The exponentiated demand equation provided an excellent fit to group data (MPT: $R^{2}=0.99$; APT: Cannabis Users $R^{2}=0.98$, Controls $R^{2}=0.98$ ) as well as individual curves (MPT: median $R^{2}=0.95$, $\mathrm{IQR}=0.91-0.97 ;$ APT: median $R^{2}=0.87$, IQR $=0.79-0.93$ ). No between-group differences were observed for alcohol AUC $\left(t_{85}=0.39, p=0.70\right)$. Similarly, no differences were observed for alcohol demand intensity $\left(t_{85}=0.50, p=0.62\right)$ or elasticity $\left(t_{85}=0.51, p=0.61\right)$. No changes in the magnitude or significance of these comparisons between cannabis users and controls were observed in covariate analyses.
Table 2

Drug Behavioral Economic Demand and Delay Discounting in Cannabis Users and Controls.

\begin{tabular}{|c|c|c|c|c|c|}
\hline \multirow[t]{2}{*}{ Variable } & \multicolumn{2}{|c|}{ Cannabis User $(\mathrm{N}=64)$} & \multicolumn{2}{|c|}{ Control $(\mathrm{N}=72)$} & \multirow[b]{2}{*}{$p$} \\
\hline & Mean (SD) & $\mathrm{n}$ & Mean (SD) & $\mathrm{n}$ & \\
\hline \multicolumn{6}{|c|}{ Demand (AUC) } \\
\hline Cannabis & $0.04(0.08)$ & 55 & - & - & - \\
\hline Alcohol & $0.02(0.02)$ & 50 & $0.02(0.01)$ & 37 & 0.70 \\
\hline \multicolumn{6}{|c|}{ Demand $\left(Q_{0}\right)$} \\
\hline Cannabis & $35.6(32.5)$ & 55 & - & - & - \\
\hline Alcohol & $7.0(8.7)$ & 50 & $7.7(16.8)$ & 37 & 0.62 \\
\hline \multicolumn{6}{|c|}{ Demand $(\alpha)$} \\
\hline Cannabis & $0.028(0.047)$ & 55 & - & - & - \\
\hline Alcohol & $0.061(0.138)$ & 50 & $0.027(0.047)$ & 37 & 0.61 \\
\hline \multicolumn{6}{|c|}{ Delay Discounting $(k)$} \\
\hline Money & $0.02(0.06)$ & 64 & $0.02(0.05)$ & 72 & 0.60 \\
\hline Cannabis & $0.97(3.79)$ & 64 & - & - & - \\
\hline Alcohol & $3.83(7.97)$ & 59 & $3.90(8.59)$ & 43 & 0.70 \\
\hline
\end{tabular}

Note. AUC $=$ area under the curve from purchase task data; $\mathrm{Q}_{0}=$ demand intensity from the exponentiated demand equation; $\alpha=$ demand elasticity from the exponentiated demand equation; $k=$ delay discounting rate from 5 -trial adjusting delay discounting task. Raw values are presented for descriptive purposes; all data were log-transformed for normality prior to analysis.

\subsection{Drug use prediction in cannabis users}

\subsubsection{Bivariate relationships}

Bivariate relationships among drug demand, delay discounting variables, and self-reported drug use in cannabis users are presented in Table 3. Cannabis AUC was significantly and positively related to grams of cannabis used per week $(r=0.45, p=0.001)$, percentage past month use days ( $r=0.31, p=0.02)$, and number of CD symptoms endorsed $(r=0.42, p=0.001)$. Similar bivariate associations were observed for cannabis demand intensity and elasticity (Supplemental Table 1). Cannabis delay discounting was related to grams of cannabis used per week $(r=0.38, p=0.002)$ and number of $\mathrm{CD}$ symptoms endorsed $(r=0.44, p \leq 0.001)$.

Intercorrelations between delay discounting and AUC revealed three significant associations all involving cannabis delay discounting. Cannabis delay discounting was significantly and positively related to cannabis demand $(r=0.34, p=0.01)$, monetary delay discounting $(r=0.42, p=0.001)$, and alcohol delay discounting $(r=0.33$, $p=0.01)$.

\subsubsection{Regression models}

Results from three regression models evaluating the unique prediction of cannabis use by AUC and delay discounting variables are presented in Table 4. Cannabis delay discounting was uniquely associated with the number of $\mathrm{CD}$ symptoms endorsed $\left(s r^{2}=0.16\right)$. The model predicting percentage of past month use days indicated a significant independent effect of cannabis AUC $\left(s r^{2}=0.09\right)$, but not alcohol AUC or delay discounting variables. A significant unique effect of cannabis AUC was also observed in the model predicting grams of cannabis used per week $\left(s r^{2}=0.12\right)$. Models including additional covariates (e.g., age, cigarette use) revealed outcomes similar in magnitude and significance. Cannabis AUC by delay discounting interactions were not significant when tested.

Additional models were used to determine if the association between cannabis use behaviors and behavioral economic demand was related to demand intensity $\left(Q_{0}\right)$ and/or elasticity $(\alpha)$. Models including demand intensity and elasticity revealed a similar pattern of effects as the AUC analysis, with cannabis demand intensity uniquely contributing to cannabis use frequency and quantity and cannabis delay discounting uniquely contributing to use severity (Table 5). Specifically, cannabis demand intensity was significantly and positively related to use quantity $\left(s r^{2}=0.29\right)$ and frequency $\left(s r^{2}=0.19\right)$. Alcohol 


\section{Cannabis}
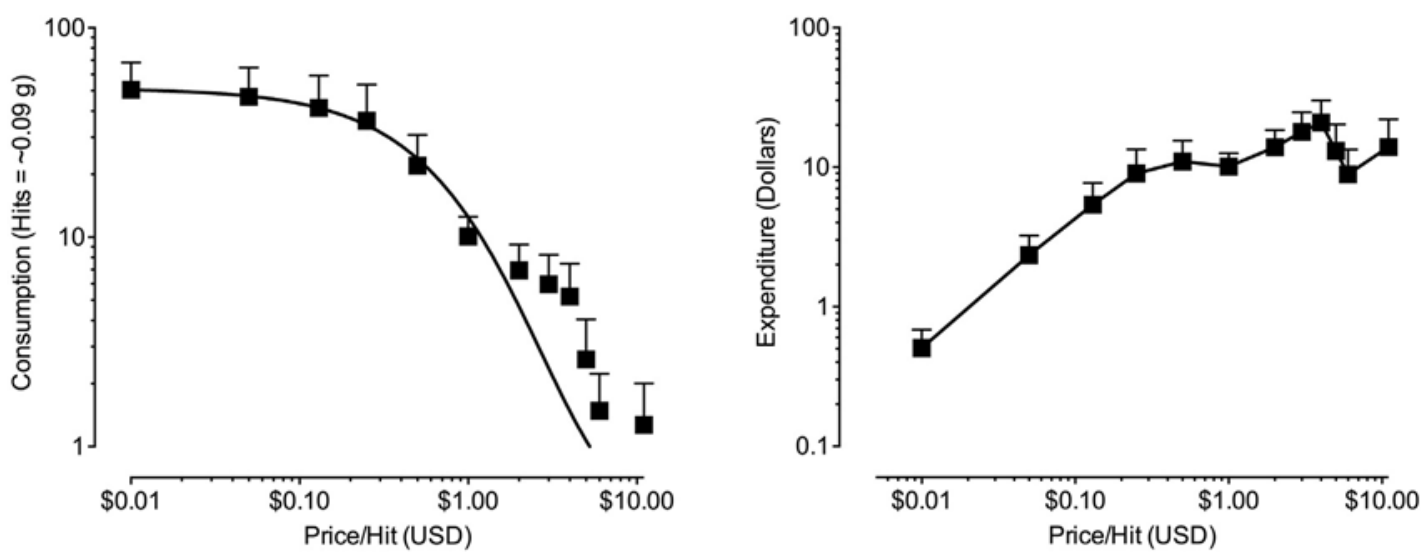

\section{Alcohol}
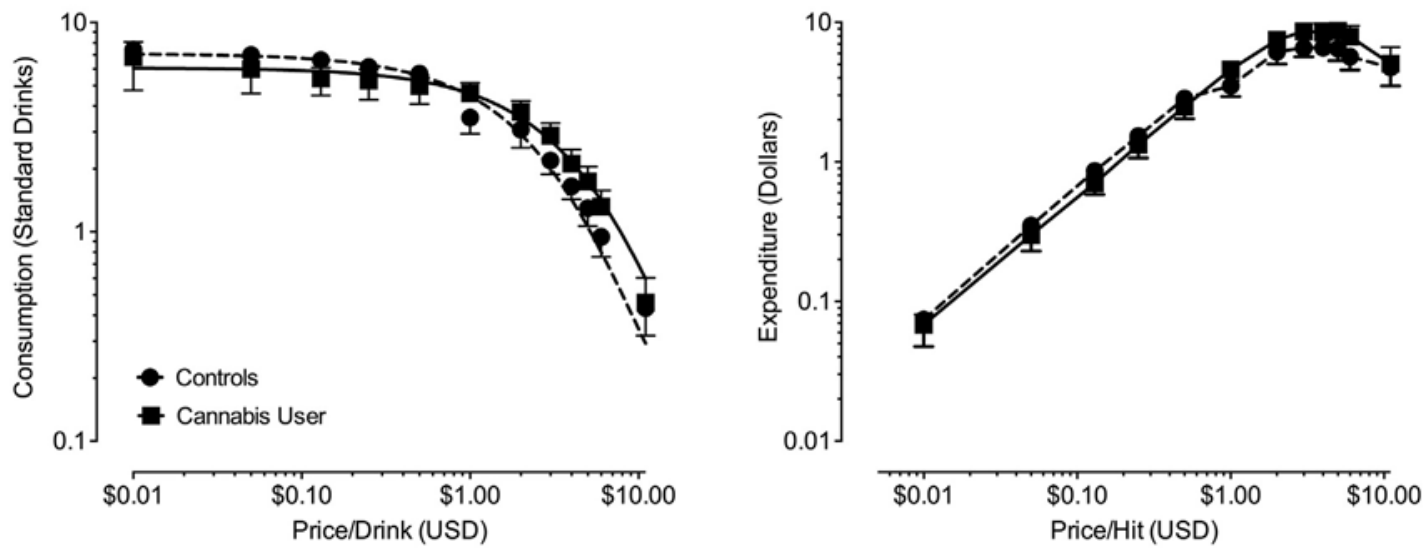

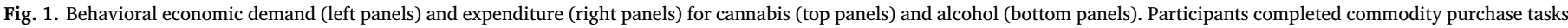

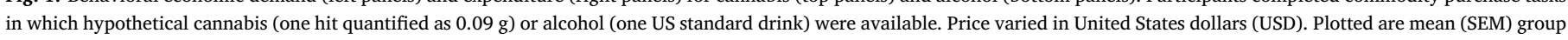

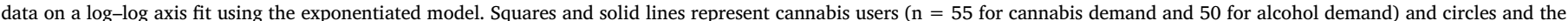
dotted lines represent controls ( $n=37$ for alcohol demand).

demand intensity also showed an inverse relationship with grams of cannabis used per week $\left(s r^{2}=0.15\right)$.

\section{Discussion}

The primary finding of the present study was that cannabis delay discounting uniquely predicted use severity (i.e., CD symptom count), whereas cannabis demand uniquely predicted use frequency (i.e., past month use days) and quantity (i.e. grams used per week) in regression models. Follow-up analyses indicated that the primary behavioral mechanism contributing to the relationship between behavioral economic demand and cannabis use frequency and quantity was demand

Table 3

Correlations Among Behavioral Economic Demand, Delay Discounting, and Drug Use Variables.

\begin{tabular}{|c|c|c|c|c|c|c|c|c|c|c|c|c|}
\hline & \multirow[b]{2}{*}{ Age } & \multirow[b]{2}{*}{ Male } & \multirow[b]{2}{*}{ Income } & \multirow[b]{2}{*}{ Alcohol Heavy } & \multicolumn{3}{|c|}{ Cannabis Use } & \multicolumn{2}{|c|}{ Demand } & \multicolumn{3}{|c|}{ Delay Discounting } \\
\hline & & & & & CD Count & Grams/Week & Month Use & CAN & ALC & MON & CAN & ALC \\
\hline \multicolumn{13}{|l|}{ Demand (AUC) } \\
\hline Cannabis $(\mathrm{n}=55)$ & 0.02 & -0.01 & 0.10 & 0.08 & $0.42^{k n}$ & $0.45^{* k}$ & $0.31^{*}$ & - & - & - & - & - \\
\hline Alcohol $(n=50)$ & 0.07 & -0.14 & 0.04 & $0.38^{* *}$ & 0.02 & 0.00 & 0.11 & 0.25 & - & - & - & - \\
\hline \multicolumn{13}{|l|}{ Delay Discounting $(k)$} \\
\hline Money $(n=64)$ & -0.16 & -0.11 & -0.22 & -0.03 & $0.27^{*}$ & 0.24 & 0.10 & 0.20 & 0.15 & - & - & - \\
\hline Cannabis $(n=64)$ & 0.01 & 0.06 & -0.07 & -0.03 & $0.44^{k * *}$ & $0.38^{* * *}$ & 0.04 & $0.34^{*}$ & 0.22 & $0.42^{* * *}$ & - & - \\
\hline Alcohol $(\mathrm{n}=59)$ & 0.04 & -0.01 & -0.02 & -0.17 & 0.12 & 0.07 & -0.10 & -0.08 & 0.01 & 0.18 & $0.33^{*}$ & - \\
\hline
\end{tabular}

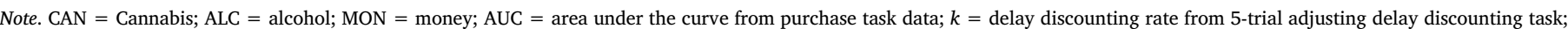

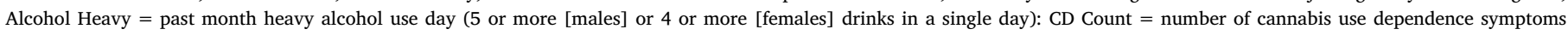

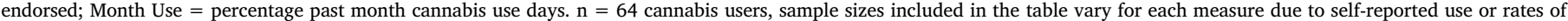
systematic data. Bold $=$ significant at $p<0.05$

$* p<0.05$.

$* * p<0.01$ 
Table 4

Area Under the Demand Curve and Delay Discounting as Predictors of Cannabis Use Variables.

\begin{tabular}{|c|c|c|c|c|c|c|c|c|c|c|c|c|}
\hline \multirow[b]{2}{*}{ Predictor } & \multicolumn{4}{|c|}{ \# CD Symptoms } & \multicolumn{4}{|c|}{ Grams Cannabis/Week } & \multicolumn{4}{|c|}{ \% Month Use Days } \\
\hline & $b$ & $\beta$ & $s r^{2}$ & $p$ & $b$ & $\beta$ & $s r^{2}$ & $p$ & $b$ & $\beta$ & $s r^{2}$ & $p$ \\
\hline Money- $k$ & 0.50 & 0.19 & 0.03 & 0.18 & 0.81 & 0.12 & 0.01 & 0.46 & 0.03 & 0.06 & $<0.01$ & 0.69 \\
\hline Cannabis-AUC & 0.77 & 0.21 & 0.03 & 0.15 & 3.70 & 0.39 & 0.12 & 0.02 & 0.23 & 0.35 & 0.09 & 0.05 \\
\hline Cannabis- $k$ & 0.93 & 0.48 & 0.16 & $0.003^{* *}$ & 0.18 & 0.04 & $<0.01$ & 0.84 & -0.08 & -0.22 & 0.03 & 0.22 \\
\hline Alcohol-AUC & -0.52 & -0.17 & 0.03 & 0.21 & -0.98 & -0.12 & 0.01 & 0.42 & 0.06 & 0.10 & 0.01 & 0.51 \\
\hline Alcohol-k & -0.13 & -0.10 & 0.01 & 0.50 & 0.32 & 0.09 & 0.01 & 0.58 & 0.02 & 0.08 & $<0.01$ & 0.66 \\
\hline
\end{tabular}

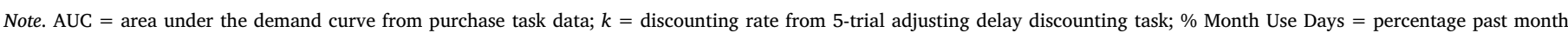
cannabis use days; \# CD Symptoms $=$ number of cannabis use dependence symptoms endorsed. $\mathrm{n}=46$ cannabis users. Bold $=$ significant at $p<0.05$

$* p<0.05$.

$* * p<0.01$.

intensity. These findings are consistent with the only other study to evaluate the unique contribution of cannabis delay discounting and demand to cannabis use behaviors (Aston et al., 2016). Several methodological concerns potentially limited the generalizability of this aforementioned study, including the use of an exclusively White sample, low prevalence of CD symptoms, and data collection following placebo cannabis self-administration that may have influenced reported delay discounting outcomes (see Metrik et al., 2009, 2012 for discussion of cannabis expectancies). Our findings suggest that these experimental parameters did not contribute to the observed relationships and that the unique contribution of delay discounting and demand to cannabis use outcomes likely generalizes to diverse experimental settings and populations. Replication studies such as this one are particularly important given recent challenges the psychological and behavioral sciences have faced regarding reproducibility (Nosek et al., 2015; Open Source Data Collection, 2015).

A consistency across online and in-laboratory samples also strengthens the case for the use of online sampling techniques to evaluate substance-using populations and behavioral mechanisms related to drug use (Koffarnus et al., 2015; Rass et al., 2015; Strickland et al., 2016a). The use of crowdsourcing comes with many benefits including increased geographic and demographic variability, targeted recruitment of hard-to-reach populations, and a relative cost and time efficiency. A requisite step for using crowdsourcing, however, is the validation of findings across Internet and laboratory settings. This is particularly important for addiction research given that drug use cannot be biologically verified in participants online. The current study adds to the extant literature demonstrating similar outcomes across in-laboratory and online samples (e.g., Johnson et al., 2015; Strickland et al., 2016a), thereby supporting the validity of the crowdsourcing approach. These findings are particularly exciting as they offer a method by which findings can be replicated across diverse samples using sampling methods that provide complementary benefits and limitations.
In addition to replicating associations involving cannabis demand and delay discounting in this novel sample, this study extends previous findings in at least three ways. First, cannabis and alcohol demand and delay discounting measures were used to determine if the observed relationships were specific to cannabis. Alcohol was selected given the extensive literature validating the alcohol purchase task, the common use of alcohol in the general population, and the expectation that alcohol use should not uniquely associate with elevated cannabis use frequency, quantity, or severity. Cannabis demand and delay discounting specifically contributed to these cannabis use variables, even after controlling for the contribution of another common drug commodity (i.e., alcohol). Alcohol demand intensity was related to cannabis use quantity when testing demand intensity and elasticity measures; however, this relationship was not observed at the bivariate level and was smaller than the relationship involving cannabis demand intensity in the multiple regression model. Although monetary delay discounting was related to CD symptom count at the bivariate level, consistent with previous findings (Aston et al., 2016), the association was not unique or significant when controlling for the cannabis commodity relationship. These outcomes indicate that these behavioral economic relationships do not likely represent a general propensity to respond in a non-specific manner to the task requirements. Instead, they suggest that these relationships with drug use outcomes are specific to drug of interest, in this case cannabis.

Second, a quantity measure was included in addition to the frequency and severity measures previously evaluated. Similar outcomes as the frequency measure were observed, wherein cannabis demand uniquely predicted the quantity of cannabis use above and beyond other demand and delay discounting measures. This distinction is important given that cannabis use frequency and quantity represent unique dimensions of use patterns and can provide unique prediction of cannabis-related problems (Zeisser et al., 2012). Third, the recently developed 5-trial adjusting delay task was used, which allowed for

Table 5

Demand Intensity, Demand Elasticity, and Delay Discounting as Predictors of Cannabis Use Variables.

\begin{tabular}{|c|c|c|c|c|c|c|c|c|c|c|c|c|}
\hline \multirow[b]{2}{*}{ Predictor } & \multicolumn{4}{|c|}{ \# CD Symptoms } & \multicolumn{4}{|c|}{ Grams Cannabis/Week } & \multicolumn{4}{|c|}{$\%$ Month Use Days } \\
\hline & $b$ & $\beta$ & $s r^{2}$ & $p$ & $b$ & $\beta$ & $s r^{2}$ & $p$ & $b$ & $\beta$ & $s r^{2}$ & $p$ \\
\hline Money- $k$ & 0.48 & 0.18 & 0.03 & 0.21 & -0.10 & -0.02 & $<0.01$ & 0.91 & -0.02 & -0.04 & $<0.01$ & 0.82 \\
\hline Cannabis- $\mathrm{Q}_{0}$ & 1.14 & 0.24 & 0.04 & 0.12 & 8.03 & 0.66 & 0.29 & $0.001^{\text {vexkex }}$ & 0.46 & 0.53 & 0.19 & $0.003^{* * *}$ \\
\hline Cannabis- $\alpha$ & -0.35 & -0.09 & 0.01 & 0.53 & -1.57 & -0.16 & 0.02 & 0.24 & -0.09 & -0.14 & 0.01 & 0.40 \\
\hline Cannabis- $k$ & 0.87 & 0.44 & 0.13 & $0.01^{* * *}$ & -0.20 & -0.04 & $<0.01$ & 0.78 & -0.10 & -0.28 & 0.05 & 0.10 \\
\hline Alcohol-Q 0 & 0.12 & 0.03 & $<0.01$ & 0.88 & -6.64 & -0.52 & 0.15 & $0.002^{* k \hbar}$ & -0.30 & -0.33 & 0.06 & 0.09 \\
\hline Alcohol- $\alpha$ & 0.55 & 0.19 & 0.02 & 0.26 & -1.82 & -0.24 & 0.03 & 0.14 & -0.18 & -0.33 & 0.06 & 0.07 \\
\hline Alcohol-k & -0.13 & -0.10 & 0.01 & 0.49 & 0.18 & 0.05 & $<0.01$ & 0.70 & 0.01 & 0.04 & $<0.01$ & 0.78 \\
\hline
\end{tabular}

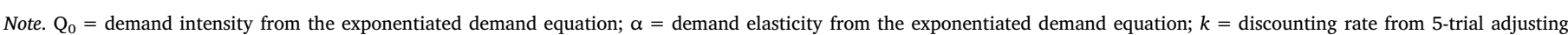

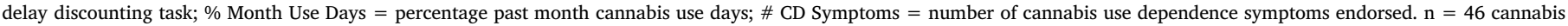
users. Bold $=$ significant at $p \leq 0.05$

$* * p<0.01$.

$* * * p<0.001$. 
rapid ( $\sim 1 \mathrm{~min}$ ) generation of delay discounting rates. Prior research has validated this task by revealing a close relationship between this rapid task and traditional adjusting delay tasks (Cox and Dallery, 2016; Koffarnus and Bickel, 2014). To our knowledge, this is the first use of drug commodities, namely alcohol and cannabis, with this rapid delay discounting task. That the relationship between monetary, alcohol, and cannabis delay discounting rates and drug use outcomes were generally significant and in the expected direction provides further support for use of this rapid assessment task in substance use research. The more complex relationships involving delay discounting (i.e., multiple regression analyses) were also consistent with previous findings (Aston et al., 2016), further supporting the validity of this delay discounting task and its future use.

Secondary analyses that focused on differences in delay discounting and demand variables between cannabis-users and non-using controls were also conducted given the limited research comparing these groups on these measures. Significant differences in monetary delay discounting as a function of cannabis use status were not observed, although this outcome is generally consistent with previous research (e.g., Johnson et al., 2010). Differences in alcohol delay discounting or demand were also not detected among cannabis users and controls. These outcomes remained after controlling for potentially important covariates, such as age or cigarette use, suggesting that the failure to detect differences was not due to low power or imbalances in relevant demographics. Taken together, these findings suggest that alterations in delay discounting and demand may not capture between-group differences in the likelihood of reporting current cannabis use. Instead, these measures may reflect differences in the propensity to use cannabis in a maladaptive or clinically relevant manner.

These findings should be considered within the context of their limitations. First, a large proportion of subjects were excluded due to inattentive or non-systematic data. This rate is generally consistent with previous mTurk research (e.g., Johnson et al., 2015) but is a limitation of conducting online research, and underscores the need to verify response fidelity due to decreased experimental control. Second, all participants completed the demand measures first followed by the delay discounting measures, and completed monetary delay discounting prior to drug tasks. Although this non-random completion order did not likely alter study outcomes, additional research would be needed for confirmation. Third, as noted above, cannabis use in the cannabis-using group was not biologically verified. However, the use of rigorous internal control measures and the consistency between this study and the previous laboratory one (Aston et al., 2016) supports the integrity of the data collected and outcomes reported. Fourth, participants were not asked about how much money they typically spent on cannabis. Because participants reported 50 or more lifetime uses, they were likely at least familiar with cannabis pricing. Fifth, the 5-trial delay discounting task did not permit examination of underlying orderliness in the raw data like traditional delay discounting tasks. However, the use of rigorous manipulation checks to evaluate participant attention and response fidelity helped to offset this limitation. Despite these potential limitations, this study replicates and extends previous research uniquely relating delay discounting and behavioral economic demand variables with cannabis use. This study also demonstrates the ability of online crowdsourcing to complement standard human laboratory procedures, thereby enhancing the overall rigor of research methods used to examine behavioral mechanisms of drug use disorders.

\section{Funding}

This work was supported by the Psi Chi Psychology Honor Society and National Science Foundation [grant number 1247392]. These funding sources had no role in study design, data collection or analysis, or preparation or submission of the manuscript.

\section{Conflicts of interest}

The authors have no real, potential, or apparent conflicts of interest to disclose that are relevant to this research.

\section{Appendix A. Supplementary data}

Supplementary data associated with this article can be found, in the online version, at http://dx.doi.org/10.1016/j.beproc.2017.03.017.

\section{References}

Amlung, M., MacKillop, J., 2015. Further evidence of close correspondence for alcohol demand decision making for hypothetical and incentivized rewards. Behav. Processes 113, 187-191.

Amlung, M., McCarty, K.N., Morris, D.H., Tsai, C.L., McCarthy, D.M., 2015a. Increased behavioral economic demand and craving for alcohol following a laboratory alcohol challenge. Addiction 110, 1421-1428.

Amlung, M., Yurasek, A., McCarty, K.N., MacKillop, J., Murphy, J.G., 2015b. Area under the curve as a novel metric of behavioral economic demand for alcohol. Exp. Clin. Psychopharmacol. 23, 168-175.

Amlung, M., Vedelago, L., Acker, J., Balodis, I., MacKillop, J., 2017. Steep delay discounting and addictive behavior: a meta-analysis of continuous associations. Addiction 112, 51-62.

Aston, E.R., Metrik, J., MacKillop, J., 2015. Further validation of a marijuana purchase task. Drug Alcohol Depend. 152, 32-38.

Aston, E.R., Metrik, J., Amlung, M., Kahler, C.W., MacKillop, J., 2016. Interrelationships between marijuana demand and discounting of delayed rewards: convergence in behavioral economic methods. Drug Alcohol Depend. 169, 141-147.

Bickel, W.K., Odum, A.L., Madden, G.J., 1999. Impulsivity and cigarette smoking: delay discounting in current never, and ex-smokers. Psychopharmacology (Berl.) 146, 447-454.

Bickel, W.K., Marsch, L.A., Carroll, M.E., 2000. Deconstructing relative reinforcing efficacy and situating the measures of pharmacological reinforcement with behavioral economics: a theoretical proposal. Psychopharmacology (Berl.) 153 44-56.

Bickel, W.K., Jarmolowicz, D.P., Mueller, E.T., Gatchalian, K.M., 2011. The behavioral economics and neuroeconomics of reinforcer pathologies: implications for etiology and treatment of addiction. Curr. Psychiatry Rep. 13, 406-415.

Bickel, W.K., Jarmolowicz, D.P., Mueller, E.T., Koffarnus, M.N., Gatchalian, K.M., 2012. Excessive discounting of delayed reinforcers as a trans-disease process contributing to addiction and other disease-related vulnerabilities: emerging evidence. Pharmacol. Ther. 134, 287-297.

Bidwell, L.C., Metrik, J., McGeary, J., Palmer, R.H., Francazio, S., Knopik, V.S., 2013. Impulsivity, variation in the cannabinoid receptor (CNR1) and fatty acid amide hydrolase (FAAH) genes, and marijuana-related problems. J. Stud. Alcohol Drugs 74, $867-878$.

Bruner, N.R., Johnson, M.W., 2014. Demand curves for hypothetical cocaine in cocainedependent individuals. Psychopharmacology (Berl.) 231, 889-897.

Bujarski, S., MacKillop, J., Ray, L.A., 2012. Understanding naltrexone mechanism of action and pharmacogenetics in Asian Americans via behavioral economics: a preliminary study. Exp. Clin. Psychopharmacol. 20, 181-190.

Center for Behavioral Health Statistics and Quality, 2016. Behavioral health trends in the United States: Results from the 2015 National Survey on Drug Use and Health (HHS Publication No. SMA 15-4927 NSDUH Series H-50). Retrieved from. http://www. samhsa.gov/data/.

Chivers, L.L., Higgins, S.T., 2012. Some observations from behavioral economics for consideration in promoting money management among those with substance use disorders. Am. J. Drug Alcohol Abuse 38, 8-19.

Collins, R.L., Vincent, P.C., Yu, J., Liu, L., Epstein, L.H., 2014. A behavioral economic approach to assessing demand for marijuana. Exp. Clin. Psychopharmacol. 22, 211-221.

Cox, D.J., Dallery, J., 2016. Effects of delay and probability combinations on discounting in humans. Behav. Processes 131, 15-23.

Green, L., Myerson, J., 2004. A discounting framework for choice with delayed and probabilistic rewards. Psychol. Bull. 130, 769-792.

Heinz, A.J., Peters, E.N., Boden, M.T., Bonn-Miller, M.O., 2013. A comprehensive examination of delay discounting in a clinical sample of Cannabis-dependent military veterans making a self-guided quit attempt. Exp. Clin. Psychopharmacol. 21, 55-65.

Hursh, S.R., 1984. Behavioral economics. J. Exp. Anal. Behav. 42, 435-452.

Hursh, S.R., 1993. Behavioral economics of drug self-administration: an introduction. Drug Alcohol Depend. 33, 165-172.

Jacobs, E.A., Bickel, W.K., 1999. Modeling drug consumption in the clinic using simulation procedures: demand for heroin and cigarettes in opioid-dependent outpatients. Exp. Clin. Psychopharmacol. 7, 412-426.

Johnson, M.W., Bickel, W.K., 2006. Replacing relative reinforcing efficacy with behavioral economic demand curves. J. Exp. Anal. Behav. 85, 73-93.

Johnson, P.S., Johnson, M.W., 2014. Investigation of bath salts use patterns within an online sample of users in the United States. J. Psychoact. Drugs 46, 369-378.

Johnson, M.W., Bickel, W.K., Baker, F., 2007. Moderate drug use and delay discounting: a comparison of heavy light, and never smokers. Exp. Clin. Psychopharmacol. 15, 187-194. 
Johnson, M.W., Bickel, W.K., Baker, F., Moore, B.A., Badger, G.J., Budney, A.J., 2010. Delay discounting in current and former marijuana-dependent individuals. Exp. Clin. Psychopharmacol. 18, 99-107.

Johnson, P.S., Herrmann, E.S., Johnson, M.W., 2015. Opportunity costs of reward delays and the discounting of hypothetical money and cigarettes. J. Exp. Anal. Behav. 103, 87-107.

Koffarnus, M.N., Bickel, W.K., 2014. A 5-trial adjusting delay discounting task: accurate discount rates in less than one minute. Exp. Clin. Psychopharmacol. 22, 222-228.

Koffarnus, M.N., Jarmolowicz, D.P., Mueller, E.T., Bickel, W.K., 2013. Changing delay discounting in the light of the competing neurobehavioral decision systems theory: a review. J. Exp. Anal. Behav. 99, 32-57.

Koffarnus, M.N., Franck, C.T., Stein, J.S., Bickel, W.K., 2015. A modified exponentia behavioral economic demand model to better describe consumption data. Exp. Clin. Psychopharmacol. 23, 504-512.

Kollins, S.H., 2003. Delay discounting is associated with substance use in college students. Addict. Behav. 28, 1167-1173.

Krishnan-Sarin, S., Reynolds, B., Duhig, A.M., Smith, A., Liss, T., McFetridge, A., Cavallo, D.A., Carroll, K.M., Potenza, M.N., 2007. Behavioral impulsivity predicts treatment outcome in a smoking cessation program for adolescent smokers. Drug Alcohol Depend. 88, 79-82.

MacKillop, J., Kahler, C.W., 2009. Delayed reward discounting predicts treatment response for heavy drinkers receiving smoking cessation treatment. Drug Alcoho Depend. 104, 197-203.

MacKillop, J., Murphy, J.G., 2007. A behavioral economic measure of demand for alcohol predicts brief intervention outcomes. Drug Alcohol Depend. 89, 227-233.

MacKillop, J., Murphy, J.G., Ray, L.A., Eisenberg, D.T., Lisman, S.A., Lum, J.K., Wilson, D.S., 2008. Further validation of a cigarette purchase task for assessing the relative reinforcing efficacy of nicotine in college smokers. Exp. Clin. Psychopharmacol. 16, $57-65$.

MacKillop, J., Amlung, M.T., Few, L.R., Ray, L.A., Sweet, L.H., Munafo, M.R., 2011. Delayed reward discounting and addictive behavior: a meta-analysis. Psychopharmacology (Berl.) 216, 305-321.

MacKillop, J., 2016. The behavioral economics and neuroeconomics of alcohol use disorders. Alcohol Clin. Exp. Res. 40, 672-685.

McClure, E.A., Vandrey, R.G., Johnson, M.W., Stitzer, M.L., 2013. Effects of varenicline on abstinence and smoking reward following a programmed lapse. Nicotine Tob. Res. 15, 139-148.

Metrik, J., Rohsenow, D.J., Monti, P.M., McGeary, J., Cook, T.A., de Wit, H., Haney, M., Kahler, C.W., 2009. Effectiveness of a marijuana expectancy manipulation: piloting the balanced-placebo design for marijuana. Exp. Clin. Psychopharmacol. 17, 217-225.

Metrik, J., Kahler, C.W., Reynolds, B., McGeary, J.E., Monti, P.M., Haney, M., de Wit, H., Rohsenow, D.J., 2012. Balanced placebo design with marijuana: pharmacological and expectancy effects on impulsivity and risk taking. Psychopharmacology (Berl.) 223, 489-499.

Metrik, J., Aston, E.R., Kahler, C.W., Rohsenow, D.J., McGeary, J.E., Knopik, V.S., MacKillop, J., 2016. Cue-elicited increases in incentive salience for marijuana Craving, demand, and attentional bias. Drug Alcohol Depend. 167, 82-88.

Murphy, J.G., MacKillop, J., 2006. Relative reinforcing efficacy of alcohol among college student drinkers. Exp. Clin. Psychopharmacol. 14, 219-227.

Murphy, J.G., MacKillop, J., Skidmore, J.R., Pederson, A.A., 2009. Reliability and validity of a demand curve measure of alcohol reinforcement. Exp. Clin. Psychopharmacol. 17, 396-404.

Murphy, J.G., Dennhardt, A.A., Yurasek, A.M., Skidmore, J.R., Martens, M.P., MacKillop,
J., McDevitt-Murphy, M.E., 2015. Behavioral economic predictors of brief alcohol intervention outcomes. J. Consult. Clin. Psychol. 83, 1033-1043.

Nosek, B.A., Alter, G., Banks, G.C., Borsboom, D., Bowman, S.D., Breckler, S.J., Buck, S., Chambers, C.D., Chin, G., Christensen, G., Contestabile, M., Dafoe, A., Eich, E., Freese, J., Glennerster, R., Goroff, D., Green, D.P., Hesse, B., Humphreys, M., Ishiyama, J., Karlan, D., Kraut, A., Lupia, A., Mabry, P., Madon, T.A., Malhotra, N., Mayo-Wilson, E., McNutt, M., Miguel, E., Paluck, E.L., Simonsohn, U., Soderberg, C., Spellman, B.A., Turitto, J., VandenBos, G., Vazire, S., Wagenmakers, E.J., Wilson, R., Yarkoni, T., 2015. Promoting an open research culture. Science 348, 1422-1425.

Odum, A.L., 2011. Delay discounting: I'm a k, you're a k. J. Exp. Anal. Behav. 96, 427-439.

Open Science Collaboration, 2015. Estimating the reproducibility of psychological science. Science 349, aac4716.

Peters, E.N., Petry, N.M., Lapaglia, D.M., Reynolds, B., Carroll, K.M., 2013. Delay discounting in adults receiving treatment for marijuana dependence. Exp. Clin. Psychopharmacol. 21, 46-54.

Petry, N.M., 2001. Delay discounting of money and alcohol in actively using alcoholics currently abstinent alcoholics, and controls. Psychopharmacology (Berl.) 154, 243-250.

Pickover, A.M., Messina, B.G., Correia, C.J., Garza, K.B., Murphy, J.G., 2016. A behavioral economic analysis of the nonmedical use of prescription drugs among young adults. Exp. Clin. Psychopharmacol. 24, 38-47.

Rachlin, H., Green, L., 1972. Commitment, choice and self-control. J. Exp. Anal. Behav. $17,15-22$.

Rass, O., Pacek, L.R., Johnson, P.S., Johnson, M.W., 2015. Characterizing use patterns and perceptions of relative harm in dual users of electronic and tobacco cigarettes. Exp. Clin. Psychopharmacol. 23, 494-503.

Sheehan, D.V., Lecrubier, Y., Sheehan, K.H., Amorim, P., Janavs, J., Weiller, E., Hergueta, T., Baker, R., Dunbar, G.C., 1998. The Mini-International Neuropsychiatric Interview (M.I.N.I.): the development and validation of a structured diagnostic psychiatric interview for DSM-IV and ICD-10. J. Clin. Psychiatry 59 (Suppl. 20), 22-23 (quiz 34-57).

Stanger, C., Ryan, S.R., Fu, H., Landes, R.D., Jones, B.A., Bickel, W.K., Budney, A.J., 2012 Delay discounting predicts adolescent substance abuse treatment outcome. Exp. Clin. Psychopharmacol. 20, 205-212.

Stein, J.S., Koffarnus, M.N., Snider, S.E., Quisenberry, A.J., Bickel, W.K., 2015. Identification and management of nonsystematic purchase task data: toward best practice. Exp. Clin. Psychopharmacol. 23, 377-386.

Strickland, J.C., Bolin, B.L., Lile, J.A., Rush, C.R., Stoops, W.W., 2016a. Differential sensitivity to learning from positive and negative outcomes in cocaine users. Drug Alcohol Depend. 166, 61-68.

Strickland, J.C., Lile, J.A., Rush, C.R., Stoops, W.W., 2016b. Comparing exponential and exponentiated models of drug demand in cocaine users. Exp. Clin. Psychopharmacol. 24, 447-455.

Washio, Y., Higgins, S.T., Heil, S.H., McKerchar, T.L., Badger, G.J., Skelly, J.M., Dantona, R.L., 2011. Delay discounting is associated with treatment response among cocainedependent outpatients. Exp. Clin. Psychopharmacol. 19, 243-248.

Yoon, J.H., Higgins, S.T., Heil, S.H., Sugarbaker, R.J., Thomas, C.S., Badger, G.J., 2007 Delay discounting predicts postpartum relapse to cigarette smoking among pregnant women. Exp. Clin. Psychopharmacol. 15, 176-186.

Zeisser, C., Thompson, K., Stockwell, T., Duff, C., Chow, C., Vallance, K., Ivsins, A., Michelow, W., Marsh, D., Lucas, P., 2012. A 'standard joint'? The role of quantity in predicting cannabis-related problems. Addict. Res. Theory 20, 82-92. 